\title{
A Review of Load Balancing Technique in Public Cloud Environments using Combination of Heuristic Function and Probability Clustering
}

\author{
Rahul Dongarde \\ M.E. Scholar \\ Branch: CSE \\ TIEIT, Bhopal (M.P.)
}

\author{
Devashri Deoskar \\ Guide \\ Dept: CSE \\ TIEIT, Bhopal (M.P.)
}

\author{
Amit Saxena \\ Head of Department \\ Dept: CSE \\ TIEIT, Bhopal (M.P.)
}

\begin{abstract}
The utility of public cloud environments is increases in current scenario of information technology. The performance and capacity of cloud environments enhanced the performance of information technology-based application. the major issue in cloud network is allocation of resource and execution of task. For the management of resource and task cloud computing uses load balancing technique. the load balancing technique, balance the load factor over the cloud environments. In this paper presents the review of cloud computing load balancing based on different heuristic-based function and clustering technique. the clustering technique gives the concept of dynamic load balancing. The heuristic based balancing technique used various algorithm such as genetic algorithm, particle swarm optimization and many more swarm-based algorithm.
\end{abstract}

\section{Keywords}

Cloud Computing, Load Balancing, VMs, Cluster, Heuristic.

\section{INTRODUCTION}

The procedure of load balancing experienced in two distinctive situations static and dynamic. The static balancing procedure utilized the procedure of CPU booking and some basic information looking system. the dynamic load balancing includes some additional procedure and increment the cost of balancer. Presently a day the dynamic load balancing system utilized heuristic based capacity. The heuristic based capacity swarm knowledge with the end goal of balancing. The dynamic load balancing model comprise of appropriated registering strategy for the controlling of load over the cloud condition. Presently a day people in general cloud computing condition utilized cloud segment procedure. the cloud parcel strategy, partitioned the cloud stack into serval part and afterward apply the way toward balancing. In this paper utilized the diagram hypothesis-based load balancing procedure, these methods isolate the heap in three circumstances one is under load, two is perfect circumstance lastly circumstance is over-burden. The all arranged virtual machine mapped by their aggregate limit of load. The all limit of virtual machine sharing based. The mutual load by virtual machine outline circumstance of cloud condition. The haring of diagram hub imports the heap of virtual machine for one hub to another hub. The time assignment outline space utilized the idea of lining hypothesis for the taking care of the employment as indicated by the occupation outline. All assets in mists are dynamic and adaptable. Cloud computing guarantees sharing of assets and normal foundation to offer administrations to clients, with the goal that operations address applications issues. Resource's/gadget's area is obscure to network's end client. Clients can likewise create/oversee cloud applications with the cloud making assets virtualization by keeping up/overseeing itself. $\mathrm{n}$ cloud, Load Balancing is a method to distribute workload more than at least one servers, arrange limit, hard drives, or other aggregate assets. Agent Datacenter usage rely on upon monstrous, huge processing equipment and system interchanges, which are liable to the normal dangers connected with any physical gadget, including equipment disappointment, control interferences and asset restrains in times of popularity. High caliber of load adjust will build the execution of the whole cloud. However, there is no broad technique that can adjust to all conceivable distinctive conditions. The rest of paper describe as in section II. Load balancing scenario. In section III describe related work. In section IV describe problem formulation and finally discuss conclusion \& future work in section V.

\section{LOAD BALANCING}

The employment landing example is not unsurprising and the limits of every hub in the cloud vary, for load balancing issue, workload control is critical to enhance framework execution and look after steadiness. Stack balancing plans relying upon whether the framework progression is critical can be both static and dynamic. Static plans don't utilize the framework data and are less perplexing while dynamic plans will bring extra expenses for the framework yet can change as the framework status changes. A dynamic plan is utilized here for its adaptability. The model has a primary controller and balancers to assemble and break down the data. In this manner, the dynamic control has little impact on the other working hubs. The framework status then gives a premise to picking the correct load balancing methodology [1].

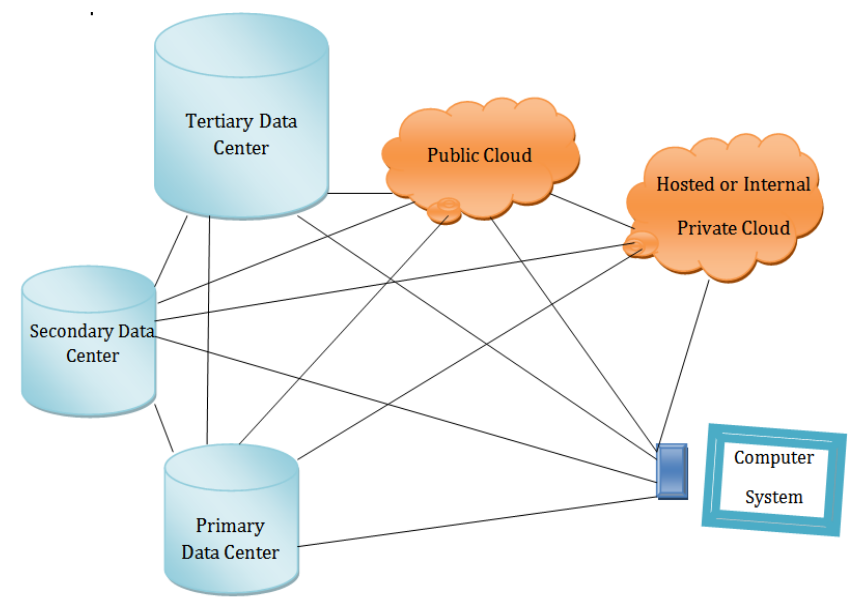

Figure 1 shows that scenario of cloud load balancing process. 


\section{RELATED WORK}

In this section discuss the related work in the field of load balancing based on different heuristic function and clustering technique.

[1] In this paper author has concentrated on the choice issue of physical hosts for conveying asked for undertakings and discussed a novel heuristic approach called Load Balancing in light of Bayes and Clustering (LB-BC). Most previous year works, for the most part, use a progression of calculations through upgrading the applicant target has inside a calculation cycle and after that selecting the ideal target hosts to accomplish the quick load adjusting impact. Be that as it may, the quick impact doesn't ensure high execution efficiency for the following undertaking despite the fact that it has capacities in accomplishing high asset usage. In light of this contention, LB-BC presents the idea of accomplishing the general load adjusting in a long haul prepare as opposed to the prompt load adjusting approaches in the present writing. LB-BC makes a restricted imperative about every single physical host planning to accomplish an undertaking sending approach with worldwide hunt ability as far as the execution capacity of registering asset.

[2] In this article writer demonstrates the discussed Load Balancing Decision Algorithm (LBDA) to oversee and adjust the heap between the virtual machines in a datacenter alongside decreasing the finishing time (Makespan) and Response time. Discoveries: The component of LBDA depends on three phases, first ascertains the VM limit and VM load to order the VMs' states (Under stacked VM, Balanced VM, High Balance VM, Overloaded). Second, ascertain the time required to execute the assignment in each VM. At last, settles on a choice to appropriate the assignments among the VMs in view of VM state and errand time required. Enhancements: They thought about the after effect of their discussed LBDA with Max-Min, Shortest Job First and Round Robin. The outcomes demonstrated that the discussed LBDA is more productive than the current calculations.

[3] In this paper, a half breed system called, Multithreaded Locality Task Scheduling and Knapsack Load Balancing (MLTS-KLB) is developed. The MLTS-KLB first calendars a few undertakings utilizing Multithreaded Locality Parallel Task Scheduling (MLPTS) calculation. The MLPTS calculation gives a definition and strategy for accomplishing bunch synchronization. Also, a Knapsack Load adjusting model is built by developing the movement-based model. At that stage, in the wake of planning the booking issues in the MLTS-KLB and presenting the MLPTS calculation-based Knapsack Fair Load Balancing calculation, the proficiency of the MLTS-KLB is approved through reproduction tests. Reenactment comes about show that the MLTS-KLB system altogether decrease the dormancy time of parallel employments and enhances the normal throughput of distributed computing condition by limiting the normal undertaking holding up time contrasted with the cutting-edge works.

[4] Author discuss to convey cloud servers at the system edge and plan the edge cloud as a tree chain of command of geoappropriated servers, in order to effectively use the cloud assets to serve the pinnacle loads from portable clients. The various leveled engineering of edge cloud empowers total of the pinnacle stacks crosswise over various levels of cloud servers to boost the measure of portable workloads being served. To guarantee efficient usage of cloud assets, they additionally discuss a workload arrangement calculation that chooses which edge cloud servers versatile projects are set on and how much computational limit is provisioned to execute each program. The execution of their discussed various leveled edge cloud engineering on serving versatile workloads is assessed by formal examination, little scale framework experimentation, and vast scale follow based reenactments.

[5] This paper concentrates the virtual machine planning of asset assignment and discussed a novel approach ECFA (Energy Conservation, Firefly Algorithm). Its primary thought is shortening the separation with ideal states through various emphases and diminishing the cost on the procedure of errand allotted to accomplish the effective scan for the ideal virtual machines. The outcomes demonstrate that ECFA accomplish the rapid scan for the virtual machines which are more qualified to perform assignments and sparing the vitality utilization of cloud server farm moderately.

[6] In this paper author show the learn about the Cloud Computing, the long-held dream of registering as an utility, can possibly change a huge piece of the IT business, making programming considerably more alluring as an administration and forming the way IT equipment is planned and bought. Engineers with inventive thoughts for new Internet benefits at no time in the future require the expansive capital costs in equipment to send their administration or the human cost to work it. They require not be worried about over-provisioning for an administration whose prevalence does not meet their expectations, along these lines squandering expensive assets, or under-provisioning for one that turns out to be fiercely prominent, in this way missing potential clients and income.

[7] In this work, author discusses a structure and a system, which measures the quality and organizes Cloud administrations. Such structure can have critical effect and will make sound rivalry among Cloud suppliers to fulfill their Service Level Agreement (SLA) and enhance their Quality of Services (QoS). Author discussed an AHP based positioning instrument which can assess the Cloud administrations in view of various applications relying upon QoS prerequisites. Their discussed system likewise addresses the test of various dimensional units of different QoS qualities by giving a uniform approach to assess relative positioning of Cloud administrations for each kind of QoS property.

[8] This paper gives an outline overview of current distributed computing structures, examines issues that present distributed computing usage has and discusses a Service-Oriented Cloud Computing Architecture (SOCCA) so mists can interoperate with each other. Besides, the SOCCA additionally discusses abnormal state plans to better support multi-occupancy highlight of distributed computing. This paper discussed an administration situated distributed computing design SOCCA that enables an application to keep running on various mists and interoperate with each other. The SOCCA is a 4-layer design that backings both SOA and distributed computing. SOCCA underpins simple application relocation starting with one cloud then onto the next and benefit redeployment to various mists by isolating the parts of administration rationale supplier and administration facilitating/cloud suppliers.

[9] In this work, author discusses a structure and a system that measures the quality and organizes Cloud administrations. Such a system can have a critical effect and will make solid rivalry among Cloud suppliers to fulfill their Service Level Agreement (SLA) and enhance their QoS. They have demonstrated the materialness of the positioning system utilizing a contextual analysis. this work shows the main structure, SIMCloud, to efficiently quantify all the QoS 
characteristics discussed by CSMIC and rank the Cloud administrations in light of these properties. They address some key difficulties by planning measurements for each quantifiable QoS property for measuring unequivocally the administration level of each Cloud supplier. Author discussed an Analytical Hierarchical Process (AHP) based positioning system which can assess the Cloud administrations in light of various applications relying upon QoS necessities.

[10] In this work author concentrate on supporting client basic leadership for choosing the most appropriate cloud design as far as infrastructural prerequisites and cost. author accomplishes this by methods for changeability demonstrating and examination systems. Right off the bat, they structure the setup space of an IaaS utilizing highlight models, generally utilized for the displaying of changeability concentrated frameworks, and present the contextual investigation of the Amazon EC2. Besides, they help the arrangement look prepare. Include models empower the utilization of different examination operations that, among others, computerize the hunt of ideal designs. Aftereffects of their examination demonstrate how their approach, with an irrelevant investigation time, beats business approaches regarding expressiveness and precision.

\section{PROBLEM FORMULATION}

The current scenario of cloud computing used various scheduling and load balancing algorithm for the improvements of allocation of resource and management of task in public cloud environments. The cluster-based cloud partition technique allocates the resource on the scenario of heavy load and ideal load in different zone of cloud scenario. The cluster technique never measures the utilization of VMs. Instead of clustering technique heuristic function play important role for the allocation of task and resource. Some common problem related to allocation technique discuss here.

$\begin{array}{ll}\text { 1. } & \text { Increase time span for job allocation [1] } \\ \text { 2. } & \text { Chain of takes [2] } \\ \text { 3. } & \text { Network traffic [3] } \\ \text { 4. } & \text { Resource utilization [4] } \\ \text { 5. } & \text { Cost of monitoring [5]. }\end{array}$

\section{CONCLUSION \& FUTURE SCOPE}

Load balancing is very important factor for the performance of public cloud network. The public cloud network has limited resource but the deployment of task rate of very high. In this paper presents the review of load balancing technique based on different heuristic technique and clustering technique. For the assessment of execution utilized cloud test system programming such is called cloud investigator. The cloud investigation programming is pack of arrangement of cloud condition and load adjusting strategy. The advancement set the assorted property of virtual machine and demand work. The characterize wellness requirements work halfway apportioned employment for devote machine and the dispersion of occupation as indicated by the procedure work scheduler.

\section{REFERENCES}

[1] Jia Zhao, Kun Yang, Xiaohui Wei, Yan Ding, Liang Hu, and Gaochao $\mathrm{Xu}$ "A Heuristic Clustering-Based Task Deployment Approach for Load Balancing Using Bayes Theorem in Cloud Environment" IEEE Transactions on Parallel And Distributed Systems, VOL. 27, 2016. Pp 305-316.

[2] Atyaf Dhari, Khaldun I. Arif "An Efficient Load Balancing Scheme for Cloud Computing" Indian Journal of Science and Technology, Vol 10, 2014. Pp 1-8.

[3] C.Antony, C.Chandrasekar "A Hybrid Multi-Threaded Task Scheduling and Knapsack Load Balancing in Multiple Cloud Centers" International Journal Of Engineering And Computer Science ISSN: 2319-7242 Volume 6 Issue 2 Feb. 2017, Page No. 20324-20332.

[4] Liang Tong, Yong Li and Wei Gao "A Hierarchical Edge Cloud Architecture for Mobile Computing" IEEE INFOCOM 2016 - The 35th Annual IEEE International Conference on Computer Communications. Pp 1-9.

[5] Yunan Zhai, Ming $\mathrm{Hu}$, and Jia Zhao "An Energyefficient Task Scheduling Approach based on Firefly Algorithm" Journal of Residuals Science \& Technology, Vol. 13, No. 8, 2016, Pp 38-384.

[6] Michael Armbrust, Armando Fox, Rean Griffith, Anthony D. Joseph, Randy Katz, Andy Konwinski, Gunho Lee, David Patterson, Ariel Rabkin, Ion Stoica, and Matei Zaharia "Above the Clouds: A Berkeley View of Cloud Computing” 2009. Pp 1-23.

[7] Saurabh Kumar Garg, Steve Versteeg and Rajkumar Buyya "SMICloud: A Framework for Comparing and Ranking Cloud Services" Pp 1-9.

[8] Wei-Tek Tsai, Xin Sun, Janaka Balasooriya "ServiceOriented Cloud Computing Architecture" Seventh International Conference on Information Technology, IEEE, 2010. Pp 684-689.

[9] Saurabh Kumar Garg, Steve Versteeg, Rajkumar Buyya "A framework for ranking of cloud computing services" Future Generation Computer Systems, 2013, 1012-1023.

[10] Jesus Garcia-Galana, Pablo Trinidada, Omer F. Ranab, Antonio Ruiz-Cortes "Automated Configuration Support for Infrastructure Migration to the Cloud" Preprint submitted to Future Generation Computer Systems, 2015. Pp 1-19. 